ARTICLE

https://doi.org/10.1038/s41467-019-13711-3

\title{
Millimetre-long transport of photogenerated carriers in topological insulators
}

\author{
Yasen Hou', Rui Wang ${ }^{2}$, Rui Xiao', Luke McClintock1, Henry Clark Travaglini', John Paulus Francia1, \\ Harry Fetsch ${ }^{3}$, Onur Erten ${ }^{4}$, Sergey Y. Savrasov ${ }^{1}$, Baigeng Wang ${ }^{5}$, Antonio Rossi, Inna Vishik (D) 1 , \\ Eli Rotenberg (i) ${ }^{6} \&$ Dong Yu (i) ${ }^{1 \star}$
}

Excitons are spin integer particles that are predicted to condense into a coherent quantum state at sufficiently low temperature. Here by using photocurrent imaging we report experimental evidence of formation and efficient transport of non-equilibrium excitons in $\mathrm{Bi}_{2-x} \mathrm{Sb}_{x} \mathrm{Se}_{3}$ nanoribbons. The photocurrent distributions are independent of electric field, indicating that photoexcited electrons and holes form excitons. Remarkably, these excitons can transport over hundreds of micrometers along the topological insulator ( $\mathrm{TI}$ ) nanoribbons before recombination at up to $40 \mathrm{~K}$. The macroscopic transport distance, combined with short carrier lifetime obtained from transient photocurrent measurements, indicates an exciton diffusion coefficient at least $36 \mathrm{~m}^{2} \mathrm{~s}^{-1}$, which corresponds to a mobility of $6 \times 10^{4} \mathrm{~m}^{2} \mathrm{~V}^{-1} \mathrm{~s}^{-1}$ at $7 \mathrm{~K}$ and is four order of magnitude higher than the value reported for free carriers in Tls. The observation of highly dissipationless exciton transport implies the formation of superfluid-like exciton condensate at the surface of Tls.

\footnotetext{
${ }^{1}$ Department of Physics, University of California, Davis, CA 95616, USA. ${ }^{2}$ Department of Physics and Astronomy, Shanghai Jiao Tong University, 200240 Shanghai, China. ${ }^{3}$ Department of Physics, Harvey Mudd College, Claremont, CA 91711, USA. ${ }^{4}$ Department of Physics, Arizona State University, Tempe, AZ 85281, USA. ${ }^{5}$ Department of Physics, Nanjing University, 210008 Jiangsu, China. ${ }^{6}$ Advanced Light Source, Lawrence Berkeley National Laboratory, Berkeley, CA 94720, USA. *email: yu@physics.ucdavis.edu
} 
A variety of systems, including double quantum wells ${ }^{1-4}$, microcavities $^{5}$, graphene $e^{6,7}$ and transition metal dichalcogenides $^{8}$, have shown signatures of exciton condensation. Dirac materials such as graphene and topological insulators (TIs) with strong Coulomb attraction and vanishing effective mass emerge as a new promising platform for achieving exciton condensate potentially at room temperature ${ }^{9-11}$. The gapless $\mathrm{TI}$ surface state is protected against backscattering and has a linear energy dispersion with massless fermions. Although freefermions have been extensively studied in TIs, much less work is carried out to understand interacting systems ${ }^{12,13}$, where electron-electron interaction may lead to emerging quasiparticles. Photoexcited electrons and holes in TIs relax to the surface Dirac cones on sub-picosecond (ps) timescales, while further carrier recombination can be much slower, ranging from a few ps to over $400 \mathrm{ps}^{14-19}$. This long-lived population inversion allows electrons and holes in the transient state to form pairs (Fig. 1a). Because of the small effective mass, excitons in Dirac materials are expected to have long de Broglie wavelength and high transition temperatures $\left(T_{\mathrm{c}}\right)^{9,10}$. The figure of merit for exciton formation in materials is $\alpha=\frac{E_{\mathrm{C}}}{E_{\mathrm{K}}}$, where $E_{\mathrm{C}}$ is the Coulomb energy and $E_{\mathrm{K}}$ is the electron kinetic energy. The linear dispersion of the TI surface state results in $\alpha=e^{2} / \epsilon \hbar v_{\mathrm{F}}$, where $e$ is the electron charge, $\epsilon$ is the dielectric constant of the material and $v_{\mathrm{F}}$ is the Fermi velocity ${ }^{20}$. The two-dimensional (2D) surface state of a three-dimensional (3D) TI, with a single nondegenerate Dirac cone, relatively low $v_{\mathrm{F}}$ (compared to graphene) and reduced $\epsilon$ at surface, has been theoretically identified as a promising candidate for realizing high- $T_{c}$ exciton condensates $^{10,11}$. In addition, the topological nature of the band structure may create exotic spin texture to the excitonic quantum state. The spin-momentum locking demands that the ground state of excitons must be a spin-triplet $p$-wave, which spontaneously breaks time reversal symmetry ${ }^{21}$.

Previous experimental evidence of exciton condensation in gapped semiconductors has been obtained from spatially resolved photoluminescence (PL) measurements, where PL images exhibit macroscopically ordered patterns ${ }^{2}$, or PL peak intensity sharply increases with reduced peak widths at lower temperature ${ }^{3,5}$. More recently, exciton formation has been experimentally demonstrated in both graphene ${ }^{6,7,22}$ and TIs ${ }^{23}$. Evidence of superfluidic excitons has also been obtained by quantum Hall drag in bilayer graphene ${ }^{6,7}$. Photocurrent imaging is a powerful experimental technique that can be applied to visualize the transport of locally photoexcited charge carriers ${ }^{24,25}$. Compared to spatially resolved $\mathrm{PL}$, it does not require materials to have strong light emission and is hence ideal to study TIs. Previous photocurrent studies of TIs have largely been on degenerately $n$-doped TIs, where photocurrent is weak with an external quantum efficiency (EQE) of $<1 \%$ and photocurrent decays rapidly as the local photoexcitation moves away from the electrical contacts to the TIs ${ }^{26-28}$.

Here, we apply scanning photocurrent microscopy (SPCM) in intrinsic 3D TIs to provide evidence on the formation of excitons and their transport of macroscopic distance.

\section{Results}

Non-local photocurrent. $\mathrm{Bi}_{2-x} \mathrm{Sb}_{x} \mathrm{Se}_{3}$ nanoribbons were grown by chemical vapour deposition $(\mathrm{CVD})^{29}$, with $x=0.38$ determined from energy-dispersive X-ray spectra. Sb doping significantly suppresses bulk conduction as evidenced by field-effect characteristics (detailed later). The experimental setup is shown in Fig. 1a, where a nanoribbon is electrically connected by two metal contacts (Fig. 1b) and is locally excited by a focused laser. As the laser beam is raster scanned on the device substrate, the photo-induced current is measured as a function of laser position and plotted into a 2D map (Fig. 1c). At room temperature, photocurrent is only observed when the laser is focused close to the contacts, caused by photo-thermoelectric effects. As the temperature is reduced, the photocurrent becomes much stronger and its direction is reversed. Importantly, the photocurrent is highly non-local and can be observed even when the laser is focused far outside the channel between the contacts. Strikingly, below $40 \mathrm{~K}$ the photocurrent barely decays even when the photoexcitation position is more than $200 \mu \mathrm{m}$ away from the contact (Fig. 1c, d). The photocurrent decay length $\left(L_{\mathrm{d}}\right)$ at various temperatures is determined by fitting photocurrent distributions with a hyperbolic function $I\left(x_{0}\right)=A \cosh \left(\frac{x_{0}-L}{L_{\mathrm{d}}}\right)$, where $x_{0}$ is the excitation position and $L$ is the length of the nanoribbon outside the channel (more details on $L_{\mathrm{d}}$ extraction and error analysis in Supplementary Notes 1 and 2). Remarkably, $L_{\mathrm{d}}$ is below $3 \mu \mathrm{m}$ at $200 \mathrm{~K}$ but increases to $0.9 \pm 0.3 \mathrm{~mm}$ at $7 \mathrm{~K}$, accompanied by an internal quantum efficiency (IQE) as high as 60\% (Fig. 1e). The observed non-local photocurrent is robust and highly reproducible in more than 10 devices measured to date.

The out-of-channel nanoribbon segment is electric field free and photoexcited carriers are expected to diffuse in this region. Photocurrent distribution in normal semiconductors free of external electric field is understood by the diffusion of minority free carriers ${ }^{24,25}$. In this model, photocurrent decays exponentially with a characteristic length of $L_{\mathrm{d}}=\sqrt{D \tau}$, where $D$ is the diffusion coefficient and $\tau$ is the lifetime of minority carriers. Transient photocurrent measurements showed $\tau=15 \pm 5 \mathrm{~ns}$ in our samples (Supplementary Fig. 6). Limited by the bandwidth of electronics, this value should be treated as an upper limit of the actual lifetime. Taking $\tau=20 \mathrm{~ns}$ and $L_{\mathrm{d}}=0.9 \mathrm{~mm}$, we estimate a lower limit of mobility $\mu=e L_{\mathrm{d}}^{2} / \tau k_{\mathrm{B}} T \approx 6 \times 10^{4} \mathrm{~m}^{2} \mathrm{~V}^{-1} \mathrm{~s}^{-1}$ at $7 \mathrm{~K}$. This is 6 orders of magnitude higher than the field-effect mobility determined in our devices $\left(\mu=0.037 \mathrm{~m}^{2} \mathrm{~V}^{-1} \mathrm{~s}^{-1}\right)$, and 4 orders of magnitude higher than the highest reported mobility in $3 \mathrm{D}$ TIs $\left(\mu \sim 1 \mathrm{~m}^{2} \mathrm{~V}^{-1} \mathrm{~s}^{-1}\right)^{30-32}$. Note that though the electron backscattering at the surface of a $3 \mathrm{D}$ TI is forbidden, scattering into other angles is possible 33,34 , resulting in finite carrier mobility. Therefore, free carrier diffusion does not explain the observation. Other photocurrent generation mechanisms, such as thermoelectric, photo-Dember effects, inhomogeneous doping and photo-recycling, are also excluded (Supplementary Note 3).

Field-independent photocurrent distributions. To understand this unusual behaviour, we performed SPCM as a function of source-drain bias $\left(V_{\mathrm{SD}}\right)$ at $7 \mathrm{~K}$ and found that the in-channel photocurrent profiles remain largely independent of $V_{\mathrm{SD}}$ (Fig. 2a, c). This is striking as $L_{\mathrm{d}}$ in normal semiconductors is expected to strongly depend on electric field, as experimentally demonstrated previously ${ }^{35-37}$. Free charge carriers move faster along the electric force, and slower against, leading to longer photocurrent decay near one contact and shorter near the other, as shown in Fig. $2 \mathrm{~d}$. Quantitatively, in this free carriers model ${ }^{38}, L_{\mathrm{d}}=\frac{2 L_{\text {diff }}^{2}}{\sqrt{L_{\text {drift }}^{2}+4 L_{\text {diff }}^{2}} \mp L_{\text {drift }}}$, where $L_{\text {diff }}=\sqrt{D \tau}, L_{\text {drift }}=\mu \tau E$ and the signs indicate $L_{\mathrm{d}}$ measured at opposite electrodes. When $E$ increases above a threshold $E_{\mathrm{c}}=\frac{k_{\mathrm{B}} T}{e L_{\text {diff }}}, L_{\mathrm{d}}$ becomes drift dominated and strongly depends on $E$. As shown in Fig. 2b, we applied $E$ up to 20 times of $E_{c}$, but the measured $L_{\mathrm{d}}$ values remained largely constant. At maximum applied field, the measured $L_{\mathrm{d}}$ value is 20 times lower than that predicted from the free carrier model. This discrepancy from the free carrier model indicates the formation of excitons. The motion of these charge neutral particles is not affected by external electric field, resulting in a $E$-independent $L_{\mathrm{d}}$ (Fig. $2 \mathrm{~b}, \mathrm{~d}$ ). Note that the applied electric field here is still much lower than that 
a

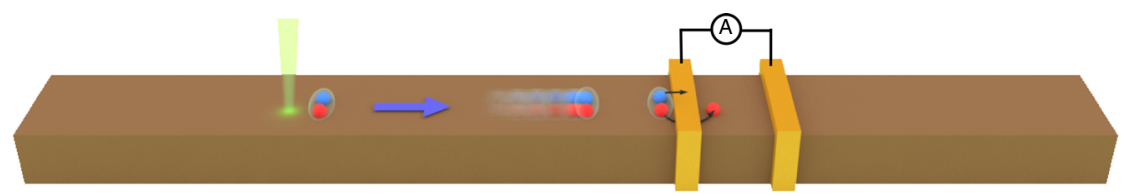

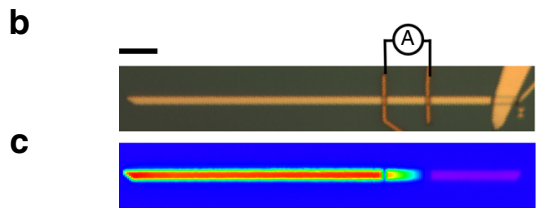

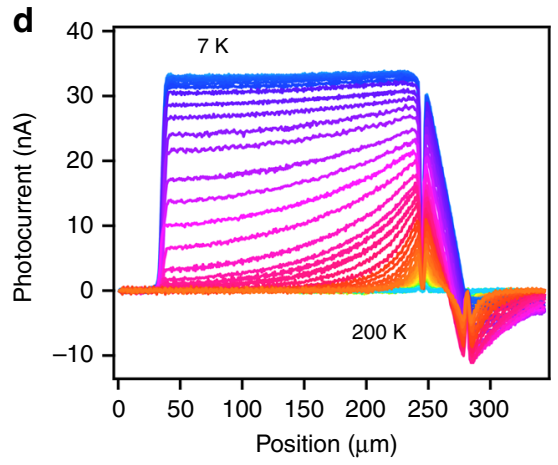

e

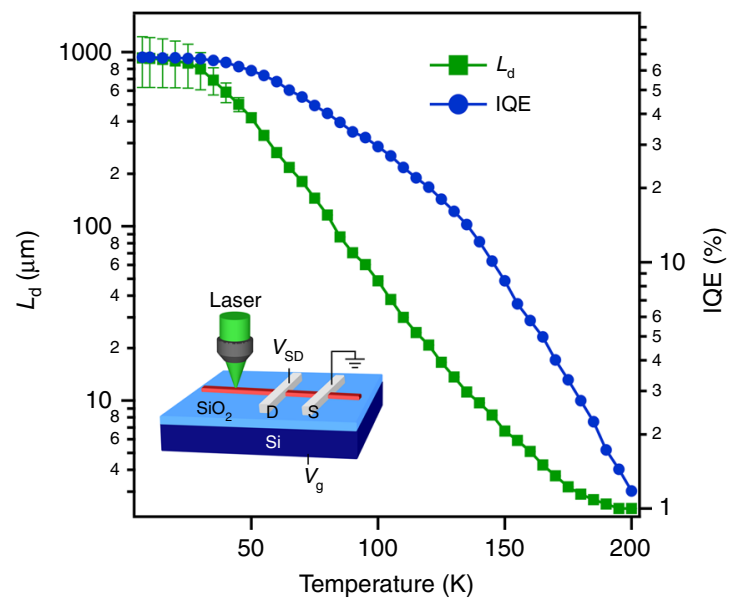

Fig. 1 Non-local photocurrent generation in a TI nanoribbon. a Schematic of exciton transport in Tls. Electrons and holes, denoted by blue and red balls, respectively, are bound and travel ballistically at $\mathrm{TI}$ surface until being separated at the metal contact. $\mathbf{b}$ Optical image of a $\mathrm{Sb}$-doped $\mathrm{Bi}_{2} \mathrm{Se}_{3} \mathrm{nanoribbon}$ $\left(305 \times 6.5 \times 0.13 \mu \mathrm{m}^{3}\right)$ in contact with $\mathrm{Cr} / \mathrm{Au}$ electrodes. The far right end of the nanoribbon is in contact with another TI nanoplate. The scale bar denotes $30 \mu \mathrm{m}$. c A photocurrent map collected by scanning a focused laser at normal incidence at $7 \mathrm{~K}$ and zero source-drain and gate biases. Laser power is 166 nW. d Photocurrent distributions along the nanoribbon axis at various temperatures. e $L_{d}$ and IQE as a function of temperature. IQE (electron collected per absorbed photon) is calculated from the photocurrent and laser power considering a reflectance of $30 \%$. The uncertainty of $L_{d}$ becomes large at low temperature because $L_{d}$ is several times larger than $L$. Inset: SPCM setup.

needed to separate excitons as estimated in Supplementary Note 4 . In addition, photoexcitation at low temperature mainly produce excitons, but with a small portion of free carriers presumably because of thermal activation, as evidenced by the small shift of the photocurrent baseline with $V_{\mathrm{SD}}$ (Supplementary Fig. 8).

Effects of Sb doping, gate, excitation wavelength and intensity. Long $L_{\mathrm{d}}$ is only observed in $\mathrm{Sb}$-doped $\mathrm{Bi}_{2} \mathrm{Se}_{3}$ samples, in which the Fermi level $\left(E_{\mathrm{F}}\right)$ is close to the Dirac point evidenced by the ambipolar gate dependence (Fig. 3d) and angle-resolved photoemission spectroscopy (ARPES) ${ }^{39}$. Micro-ARPES spectra of these nanostructures have demonstrated clear Dirac cones and indicated that the samples are slightly $n$-doped relative to the Dirac point, but with $E_{\mathrm{F}}$ below the bulk conduction band (Supplementary Fig. 2). $L_{\mathrm{d}}$ in samples with low Sb doping is shorter than that with more $\mathrm{Sb}$ (Supplementary Fig. 9). In pure $\mathrm{Bi}_{2} \mathrm{Se}_{3}$ that is degenerately $n$-doped, photocurrent with much lower magnitude is observed solely near the contacts (Fig. 3a). This explains why non-local photocurrent has not been reported in TIs, though photocurrent mapping in TIs has been studied in previous work ${ }^{26-28}$. Consistent with the doping-dependent photocurrent, we also found that $L_{\mathrm{d}}$ and IQE can be greatly modulated by gate voltage $\left(V_{\mathrm{g}}\right)$. Photocurrent first increases slightly at negative $V_{\mathrm{g}}$ when $E_{\mathrm{F}}$ is lowered closer to the Dirac point. But as $V_{\mathrm{g}}$ becomes more negative and tunes the TI from $n$-type to $p$-type (Fig. $3 \mathrm{~d}$ ), both $L_{\mathrm{d}}$ and IQE drop sharply (Fig. $4 \mathrm{a}, \mathrm{d}$ ).

The photocurrent distributions are also measured as a function of light polarization, light intensity and excitation wavelength. Both circularly and linearly polarized laser beams are applied, but the resulting photocurrent distributions are independent of the polarization due to the normal incidence of the laser used in this work ${ }^{28} . L_{\mathrm{d}}$ is found to decrease at higher laser intensity (Fig. $4 \mathrm{~b}, \mathrm{e}$ ) but is independent of excitation wavelength in a wide range of $500-1700 \mathrm{~nm}$ (Fig. 4c, f). The latter rules out the possibility of surface plasmon polariton $(\mathrm{SPP})^{40}$ since the SPP propagation length is expected to be wavelength dependent ${ }^{41,42}$. The normal incidence configuration with light injection from free space also unlikely creates surface plasmon due to momentum mismatch. Surface plasmons are expected to exist at high electron density ${ }^{40}$, but we only observed long photocurrent decay lengths in intrinsic samples. In addition, the wavelength independent $L_{\mathrm{d}}$ confirms that the second Dirac cone $1.5 \mathrm{eV}$ above the conduction band edge $^{43}$ is not involved in the exciton formation.

\section{Discussion}

One possible way to understand the observed highly efficient carrier transport at low temperature is the formation of superfluid-like exciton condensate in TIs. Different from free carriers, which suffer from scattering, excitons are bosons and can condense into a coherent quantum state at low temperature. In this picture, the photoexcitation mainly generate charge carriers in the bulk of the TI material. Then, these photoexcited carriers undergo a fast relaxation process within a few picoseconds and relax to the surface states where recombination is much slower up to hundreds of picoseconds. The electrons and holes at the surface form excitons at sufficiently low temperature. The exciton formation opens many-body energy gaps at the surface states similar to the energy gap associated with Cooper pairs in superconductors ${ }^{10,11}$. The excitons propagate across the TI surface ballistically over hundreds of micrometres. The flow of excitons does not generate an electrical current because excitons are charge neutral. However, as excitons reach the metal-TI contact, they are separated and create photocurrent. $\mathrm{Bi}_{2} \mathrm{Se}_{3}$ makes 
a

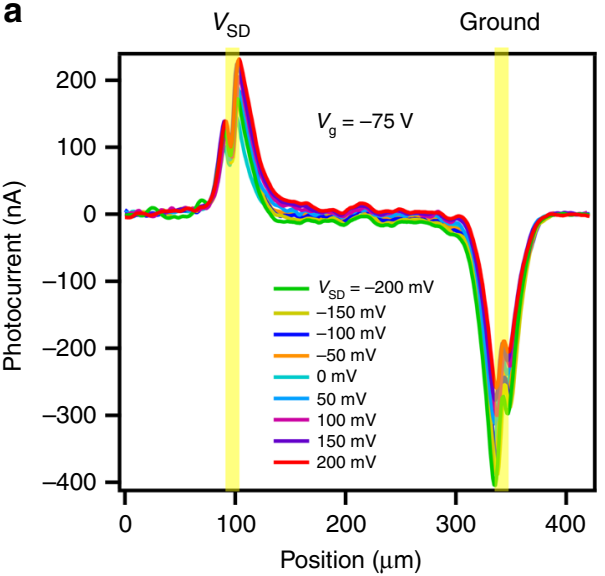

c

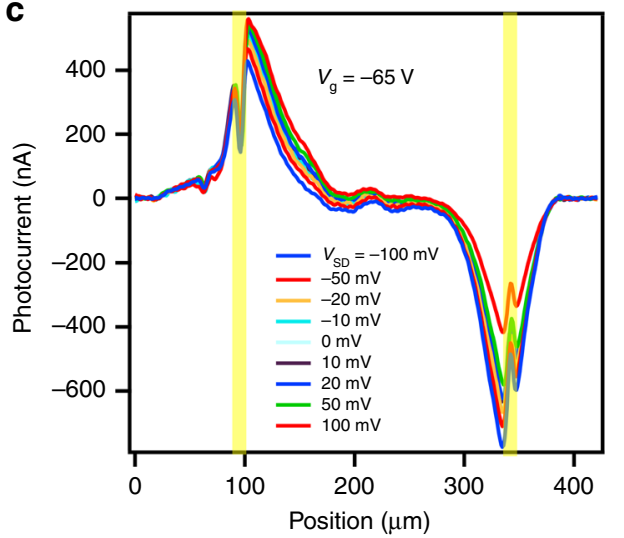

b

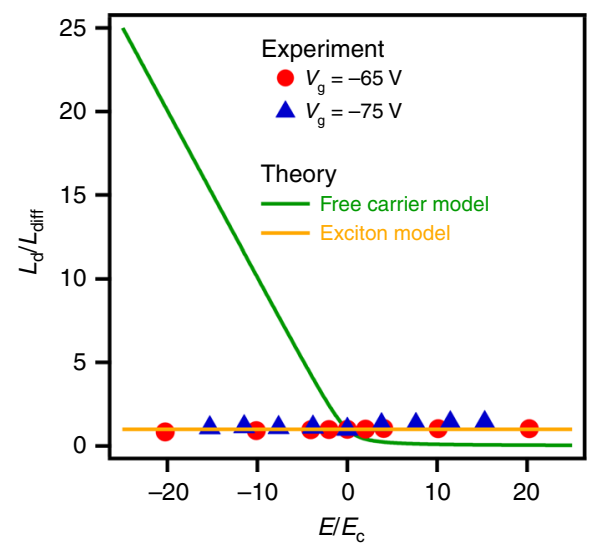

d

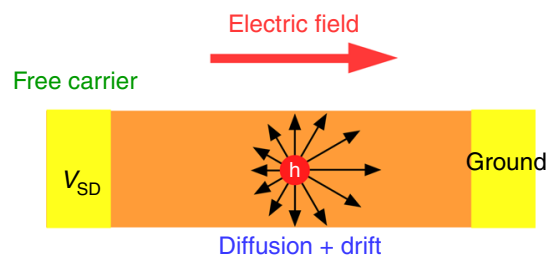

Exciton

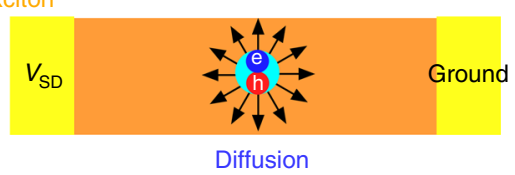

Fig. 2 Electric field-independent photocurrent profiles at $\mathbf{7} \mathbf{K}$. The dark current induced by $V_{S D}$ is subtracted from the total current. A gate voltage is applied to shorten $L_{d}$ in order to observe possible electric field induced changes. a, c Photocurrent as a function of laser excitation position along the nanoribbon axis at various $V_{S D}$ and $V_{g}=-75 \mathrm{~V}\left(L_{\text {diff }}=11.4 \mu \mathrm{m}\right)$ and $-65 \mathrm{~V}\left(L_{\text {diff }}=30.1 \mu \mathrm{m}\right)$, respectively. Laser power is $20 \mu \mathrm{W}$. $V$ ertical yellow lines indicate the contacts. $\mathbf{b} L_{d}$ near the left electrode extracted from $\mathbf{a}, \mathbf{c}$ as a function of electric field $E$. $L_{d}$ is normalized by diffusion length $L_{\text {diff }}$ measured at $V_{S D}=0 V$. $E$ is normalized by critical electric field $E_{c}$. Green and orange solid lines represent the photocurrent decay lengths predicted by the free carrier model $^{38}$ and exciton model, respectively. The field-independent $L_{d}$ indicates carriers are bound into charge neutral excitons. $\mathbf{d}$ Schematic showing that motion of free carriers is affected by external electric field, while excitons are not.

Ohmic contact to metals but strong band bending of hundreds of $\mathrm{meV}$ at the junction ${ }^{44}$ facilitates efficient charge transfer (see band diagram and equivalent circuit model in Supplementary Fig. 3). The high IQE value at low temperature indicates that a large fraction of photoexcited carriers condense in the superfluid state.

This exciton condensate model is consistent with the sensitive dependence of photocurrent distributions on temperature, doping, gate and intensity. Both $L_{\mathrm{d}}$ and IQE increase rapidly by orders of magnitude as temperature decreases and saturate below $40 \mathrm{~K}$ (Fig. 1e), which is consistent with the expectation of strong temperature dependence of Bose-Einstein condensate. This saturation temperature corresponds to $T_{\mathrm{c}}=40 \mathrm{~K}$, significantly higher than most of the previous reports of exciton condensation $^{1-6}$. Long $L_{\mathrm{d}}$ is only observed in intrinsic TIs when $E_{\mathrm{F}}$ is close to the Dirac point, as shown by doping and gate effects. The rapid drop of $L_{\mathrm{d}}$ as $V_{\mathrm{g}}$ becomes more negative (Fig. 4a, d) is likely because of faster carrier recombination caused by the mixing of surface states and bulk valence band in $p$-type TIs, as the Dirac point is close to the bulk valence band in $\mathrm{Bi}_{2} \mathrm{Se}_{3}$. Furthermore, the strong dependence of $L_{\mathrm{d}}$ on $E_{\mathrm{F}}$ indicates that excitons are at the surface of TIs. While the carrier lifetime at the TI surface sensitively depends on $E_{\mathrm{F}}$ and is over $400 \mathrm{ps}$ in intrinsic samples, the lifetime in the bulk is always short in the order of picoseconds ${ }^{14-18}$. As a result, excitons in the bulk are required to travel at a speed 2 orders of magnitude higher than the Fermi velocity in order to propagate across the $200-\mu \mathrm{m}$ nanoribbon within this lifetime, which is highly unlikely. Finally, the strong light intensity dependence indicates that stronger screening at high intensity makes exciton formation more difficult. The theoretically estimated $T_{\mathrm{c}}$ as a function of excitation power (Supplementary Note 4 ) is in good agreement with the experimental observation (Supplementary Figs. 10 and 11).

Different theories suggest that exciton condensates can be either an insulating ${ }^{45,46}$ or a superfluid state $e^{9,47,48}$. Experimentally, signatures of both excitonic insulator ${ }^{12}$ and excitonic superfluid $^{3,4,6,7}$ have been reported. The observed highly dissipationless transport of photogenerated carriers in TIs provides strong evidence supporting superfluidity. As pointed out in reference ${ }^{48}$, superfluidity in $\mathrm{He}^{4}$ or superconductors can be distinct from that in exciton condensate, where the former is via mass flow and the later is via energy flow. It is interesting to note that mass flow is not necessary for the observed long photocurrent decay. The energy flow from the photoexcitation point to electrical contact can also result in non-local photocurrent. Furthermore, Fig. 1e shows that $L_{\mathrm{d}}$ decreases gradually when temperature is increased above $T_{\mathrm{c}}$, indicating that the phase transition is not sharp. This can be understood by considering the 
a
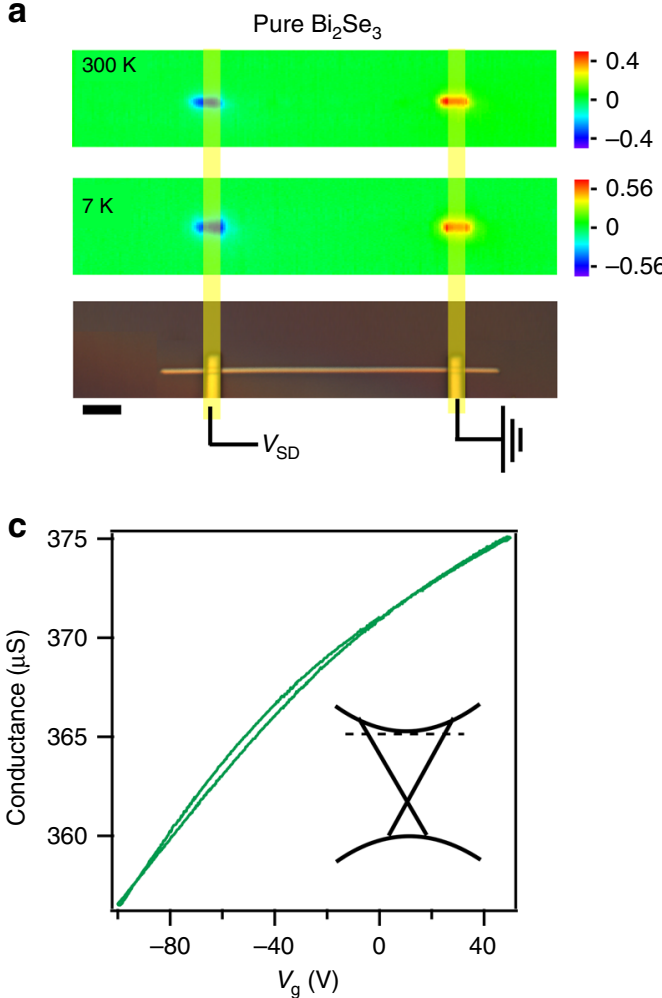

b
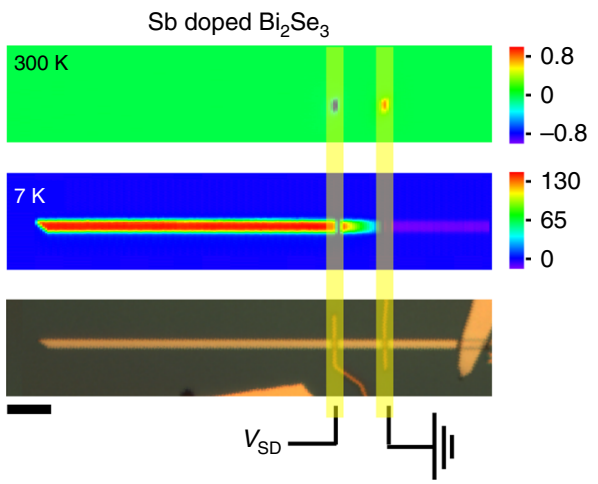

d

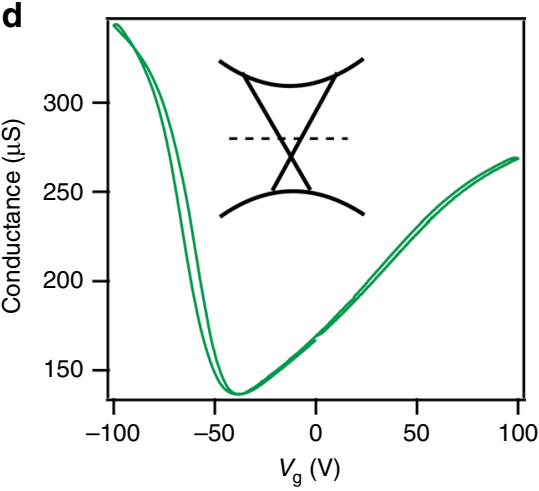

Fig. 3 Doping-dependent photocurrent profiles. a, b Photocurrent and optical images, where vertical yellow lines indicate the contacts. Colour scales are current in nanoampere. Laser power is $723 \mathrm{nW}$. c, d Gate-dependent conductance measured in the dark at $7 \mathrm{~K}$. Insets: band diagrams showing $E_{\mathrm{F}}$ positions. a, $\mathbf{c}$ are for pure $\mathrm{Bi}_{2} \mathrm{Se}_{3}$, where $E_{\mathrm{F}}$ is close to the conduction band. Field-effect mobility and electron concentration are estimated to be $\mu=329 \mathrm{~cm}^{2} \mathrm{~V}^{-1} \mathrm{~s}^{-1}$, $n=3.25 \times 10^{18} \mathrm{~cm}^{-3}$. The photocurrent is only observed when excitation is close to the contacts. $\mathbf{b}$, $\mathbf{d} \mathrm{Sb}$ doping lowers $E_{\mathrm{F}}$ as evidenced by ambipolar conduction. $\mu=371 \mathrm{~cm}^{2} \mathrm{~V}^{-1} \mathrm{~s}^{-1}, n=9.3 \times 10^{16} \mathrm{~cm}^{-3}$ for electrons. The scale bars correspond to $3 \mu \mathrm{m}$ in $\mathbf{a}$ and $30 \mu \mathrm{m}$ in $\mathbf{b}$.
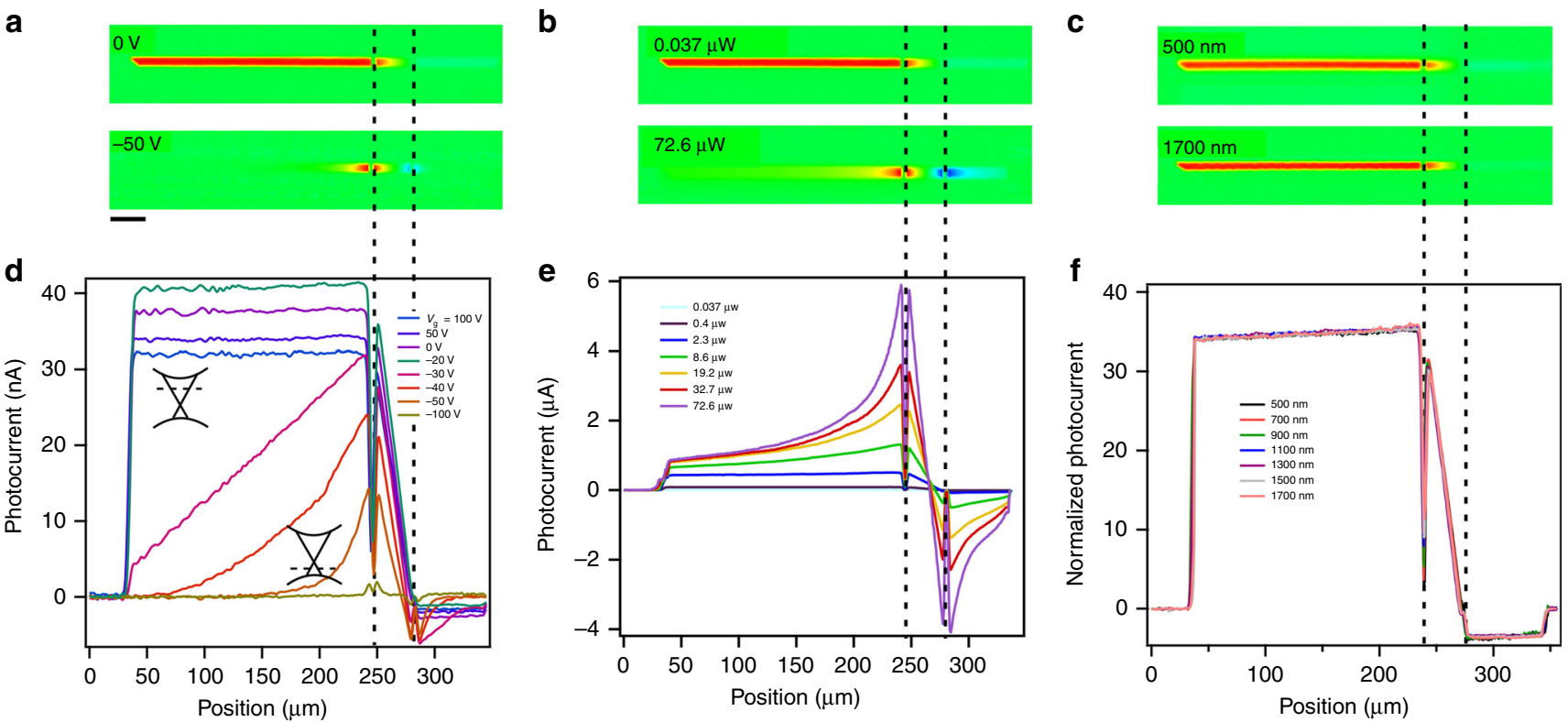

Fig. 4 Effects of wavelength, gate voltage and laser power on photocurrent decay lengths in $\mathbf{S b}$-doped $\mathbf{B i}_{2} \mathbf{S e}_{\mathbf{3}}$. The dashed lines indicate the contacts. The measurements are carried out at $7 \mathrm{~K}$ and zero source-drain and gate biases. a-c $2 \mathrm{D}$ photocurrent maps. $\mathbf{d}$-f line cuts along the nanoribbons axis. a, d Gate voltage dependence. Inset, band diagrams showing $E_{\mathrm{F}}$ position. Laser power is $166 \mathrm{nW}$. b, e Laser power dependence. c, f Wavelength dependence. Laser power from 77 to $280 \mathrm{nW}$ was used for different wavelengths to maintain the same exciton injection rate. The scale bar denotes $30 \mu \mathrm{m}$. 
Kosterliz-Thouless (KT) phase transition ${ }^{49}$, which predicts the phase transition of a $2 \mathrm{D}$ system into a superfluid under the KT transition temperature $T_{\mathrm{KT}}$, despite the absence of conventional long-range order in $2 \mathrm{D}$. In this theory, the vortex excitations emerge and become closely bound for $T<T_{\mathrm{KT}}$, resulting in a coherent state that displays frictionless exciton transport ${ }^{49,50}$. For $T>T_{\mathrm{KT}}$, the vortices are unbound and the correlation length scales exponentially with $T^{51}\left(\xi=c_{1} \exp \left[\frac{c_{2}}{T-T_{\mathrm{KT}}}\right]^{1 / 2}\right)$. The characteristic length of exciton transport, that is, $L_{\mathrm{d}}$, is then expected to have a similar scaling and decrease exponentially at higher temperature. The $T_{\mathrm{KT}}$ values estimated by the Hartree-Fock mean-field calculations are in good agreement with the experimental observation (Supplementary Fig. 11). Finally, the topological exciton condensate, as implied by the above observations, is unique in that it results from direct excitons at the TI surface, while excitons in previous systems are indirect with electrons and holes either spatially separated by an insulating layer ${ }^{1-4,6}$ or at energy minima with different momenta ${ }^{8}$. Consequently, the coherent macroscopic quantum states are robust and can be realized in simple devices that do not involve complex structures, enabling widespread applications in quantum computations and spintronics.

\section{Methods}

Nanoribbon growth and device fabrication. The CVD growth was carried out in a Lindberg Blue $\mathrm{M}$ tube furnace, following similar procedures as in previous work ${ }^{29}$. The system was first evacuated to a base pressure of $30 \mathrm{mT}$ Torr and Ar was then injected and a room pressure was maintained. For a typical growth, $116 \mathrm{mg}$ of $\mathrm{Bi}_{2} \mathrm{Se}_{3}$ powder $(99.999 \%$, Alfa Aesar) was mixed with $20-35 \mathrm{mg}$ of Sb powder (99.999\%, Alfa Aesar) and placed in a small quartz tube at the centre of the tube furnace. Se pellets $(250 \mathrm{mg})(99.999 \%$, Johnson Matthey Inc.) were placed in another quartz tube upstream by a distance of $16 \mathrm{~cm}$. A silicon substrate was placed $14 \mathrm{~cm}$ downstream from the centre of the furnace. The surface of the silicon substrate was coated with $10 \mathrm{~nm}$ of $\mathrm{Au}$ as a catalyst by electron beam evaporation. The temperature at the centre of the furnace was $680^{\circ} \mathrm{C}$, the Ar flow rate was 150 sccm (standard cubic centimetres per minute) and the growth time was $5 \mathrm{~h}$. After that, the furnace was cooled down to room temperature over $\sim 3 \mathrm{~h}$. The growth yields both nanoribbons and nanoplates (Supplementary Fig. 1a). The as-grown nanoribbons were then transferred to $300 \mathrm{~nm} \mathrm{SiO} 2$ covered Si substrates, where single nanoribbon field-effect transistor (FET) devices were fabricated using a standard electron beam lithography process. Top metal contacts $(10 \mathrm{~nm} \mathrm{Cr} / 290 \mathrm{~nm}$ $\mathrm{Au}$ or $10 \mathrm{~nm} \mathrm{Ti} / 290 \mathrm{~nm} \mathrm{Au}$ ) were made using an electron beam evaporator (CHA) or a sputterer (Lesker). A typical device is shown in Supplementary Fig. 1b, c.

Optoelectronic measurements. The low temperature measurements were performed in a cryostat (Janis ST-500). Current-voltage curves were measured through a current preamplifier (DL Instruments, model 1211) and a NI data acquisition system. SPCM measurements were performed using a home-built setup based upon an Olympus microscope. Briefly, a $532 \mathrm{~nm}$ CW laser or a tuneable laser (NKT SuperK plus) was focused by a $\times 10$ NA 0.3 objective lens to a diffraction limited spot with a size of $\sim 3 \mu \mathrm{m}$ and raster scanned on a planar nanoribbon device by a pair of mirrors mounted on galvanometers, while both reflectance and photocurrent were simultaneously recorded to produce $2 \mathrm{D}$ maps. The laser power was controlled by a set of neutral density (ND) filters and was measured by a power meter underneath the objective lens. Fast photoresponse measurements were performed using a pulsed laser (Thorlabs $450 \mathrm{~nm}$, pulse width 10-40 ns), highspeed amplifiers (Femto DHPCA-100) and a digital oscilloscope. The results in Figs. 1, 3 and 4 (except Fig. 3a, c) are obtained from one device for consistency, while the general trends are highly repeatable in more than 10 devices measured to date.

Micro-ARPES. Micro-ARPES experiments were performed at the Microscopic and Electronic Structure Observatory beamline 7.0.2 at the Advanced Light Source. Samples were removed from growth chamber, sealed in an argon gas environment and inserted into the micro-ARPES UHV end-station with a base pressure better than $5 \times 10^{-11}$ mbar via attached glovebox. ARPES data were collected at $70 \mathrm{~K}$ using a hemispherical Scienta R4000 electron analyser, $100 \mathrm{eV}$ photon energy and a beam size of $50 \mu \mathrm{m}^{2}$. A typical micro-ARPES spectrum of a Sb-doped $\mathrm{Bi}_{2} \mathrm{Se}_{3}$ nanoplate is shown in Supplementary Fig. 2.

\section{Data availability}

The data that support the findings of this study are available from the corresponding author upon reasonable request.

Received: 4 June 2019; Accepted: 21 November 2019; Published online: 16 December 2019

\section{References}

1. Spielman, I. B., Eisenstein, J. P., Pfeiffer, L. N. \& West, K. W. Resonantly enhanced tunneling in a double layer quantum Hall ferromagnet. Phys. Rev. Lett. 84, 5808-5811 (2000).

2. Butov, L. V., Gossard, A. C. \& Chemla, D. S. Macroscopically ordered state in an exciton system. Nature 418, 751-754 (2002).

3. Butov, L. V., Lai, C. W., Ivanov, A. L., Gossard, A. C. \& Chemla, D. S. Towards Bose-Einstein condensation of excitons in potential traps. Nature 417, 47-52 (2002).

4. Nandi, D., Finck, A. D. K., Eisenstein, J. P., Pfeiffer, L. N. \& West, K. W. Exciton condensation and perfect Coulomb drag. Nature 488, 481-484 (2012).

5. Balili, R., Hartwell, V., Snoke, D., Pfeiffer, L. \& West, K. Bose-Einstein condensation of microcavity polaritons in a trap. Science 316, 1007-1010 (2007).

6. Liu, X., Watanabe, K., Taniguchi, T., Halperin, B. I. \& Kim, P. Quantum Hall drag of exciton condensate in graphene. Nat. Phys. 13, 746-750 (2017).

7. Li, J. I. A., Taniguchi, T., Watanabe, K., Hone, J. \& Dean, C. R. Excitonic superfluid phase in double bilayer graphene. Nat. Phys. 13, 751 (2017).

8. Kogar, A. et al. Signatures of exciton condensation in a transition metal dichalcogenide. Science 358, 1314-1317 (2017).

9. Min, H., Bistritzer, R., Su, J.-J. \& MacDonald, A. H. Room-temperature superfluidity in graphene bilayers. Phys. Rev. B 78, 121401 (2008).

10. Triola, C., Pertsova, A., Markiewicz, R. S. \& Balatsky, A. V. Excitonic gap formation in pumped Dirac materials. Phys. Rev. B 95, 205410 (2017).

11. Pertsova, A. \& Balatsky, A. V. Excitonic instability in optically pumped threedimensional Dirac materials. Phys. Rev. B 97, 075109 (2018).

12. Du, L. et al. Evidence for a topological excitonic insulator in InAs/GaSb bilayers. Nat. Commun. 8, 1971 (2017).

13. Seradjeh, B., Moore, J. E. \& Franz, M. Exciton condensation and charge fractionalization in a topological insulator film. Phys. Rev. Lett. 103, 066402 (2009).

14. Zhu, S. et al. Ultrafast electron dynamics at the Dirac node of the topological insulator $\mathrm{Sb}_{2} \mathrm{Te}_{3}$. Sci. Rep. 5, 13213 (2015).

15. Freyse, F., Battiato, M., Yashina, L. V. \& Sánchez-Barriga, J. Impact of ultrafast transport on the high-energy states of a photoexcited topological insulator. Phys. Rev. B 98, 115132 (2018).

16. Sumida, K. et al. Prolonged duration of nonequilibrated Dirac fermions in neutral topological insulators. Sci. Rep. 7, 14080 (2017).

17. Wang, Y. H. et al. Measurement of intrinsic Dirac fermion cooling on the surface of the topological insulator $\mathrm{Bi}_{2} \mathrm{Se}_{3}$ using time-resolved and angleresolved photoemission spectroscopy. Phys. Rev. Lett. 109, 127401 (2012).

18. Hajlaoui, M. et al. Tuning a Schottky barrier in a photoexcited topological insulator with transient Dirac cone electron-hole asymmetry. Nat. Commun. 5, 3003 (2014).

19. Seifert, P., Vaklinova, K., Kern, K., Burghard, M. \& Holleitner, A. Surface state-dominated photoconduction and $\mathrm{THz}$ generation in topological $\mathrm{Bi}_{2} \mathrm{Te}_{2} \mathrm{Se}$ nanowires. Nano Lett. 17, 973-979 (2017)

20. Kotov, V. N., Uchoa, B., Pereira, V. M., Guinea, F. \& Castro Neto, A. H. Electron-electron interactions in graphene: current status and perspectives. Rev. Mod. Phys. 84, 1067-1125 (2012).

21. Wang, R., Erten, O., Wang, B. \& Xing, D. Y. Prediction of a topological p + ip excitonic insulator with parity anomaly. Nat. Commun. 10, 210 (2019).

22. Ju, L. et al. Tunable excitons in bilayer graphene. Science 358, 907-910 (2017)

23. Kung, H.-H. et al. Observation of chiral surface excitons in a topological insulator $\mathrm{Bi}_{2} \mathrm{Se}_{3}$. Proc. Natl Acad. Sci. USA 116, 4006-4011 (2019).

24. Graham, R. \& Yu, D. Scanning photocurrent micrscopy in semiconductor nanostructures. Mod. Phys. Lett. B 27, 1330018 (2013).

25. Fu, D. et al. Electrothermal dynamics of semiconductor nanowires under local carrier modulation. Nano Lett. 11, 3809-3815 (2011).

26. Kastl, C. et al. Local photocurrent generation in thin films of the topological insulator $\mathrm{Bi}_{2} \mathrm{Se}_{3}$. Appl. Phys. Lett. 101, 4 (2012).

27. Qu, D.-X. et al. Anomalous helicity-dependent photocurrent in the topological insulator $\left(\mathrm{Bi}_{0.5} \mathrm{Sb}_{0.5}\right)_{2} \mathrm{Te}_{3}$ on a GaAs substrate. Phys. Rev. B 97, 045308 (2018).

28. McIver, J. W., Hsieh, D., Steinberg, H., Jarillo-Herrero, P. \& Gedik, N. Control over topological insulator photocurrents with light polarization. Nat. Nanotechnol. 7, 96-100 (2011).

29. Kong, D. S. et al. Topological insulator nanowires and nanoribbons. Nano Lett. 10, 329-333 (2010). 
30. Butch, N. P. et al. Strong surface scattering in ultrahigh-mobility $\mathrm{Bi}_{2} \mathrm{Se}_{3}$ topological insulator crystals. Phys. Rev. B 81, 241301 (2010).

31. Xiu, F. et al. Manipulating surface states in topological insulator nanoribbons. Nat. Nanotechnol. 6, 216 (2011).

32. Ko, W. et al. Accessing the intrinsic spin transport in a topological insulator by controlling the crossover of bulk-to-surface conductance. Phys. Rev. Lett. 121, 176801 (2018)

33. Peng, X., Yang, Y., Singh, R. R. P., Savrasov, S. Y. \& Yu, D. Spin generation via bulk spin current in three-dimensional topological insulators. Nat. Commun. 7, 10878 (2016).

34. Culcer, D., Hwang, E. H., Stanescu, T. D. \& Das Sarma, S. Two-dimensional surface charge transport in topological insulators. Phys. Rev. B 82, 155457 (2010).

35. Graham, R., Miller, C., Oh, E. \& Yu, D. Electric field dependent photocurrent decay length in single lead sulfide nanowire field effect transistors. Nano Lett. 11, 717-722 (2011).

36. Gutsche, C. et al. Direct determination of minority carrier diffusion lengths at axial GaAs nanowire p-n junctions. Nano Lett. 12, 1453-1458 (2012).

37. $\mathrm{Hu}, \mathrm{X}$. et al. Visualizing carrier transport in metal halide perovskite nanoplates via electric field modulated photoluminescence imaging. Nano Lett. 18, 3024-3031 (2018).

38. Ryvkin, S. M. Photoelectric Effects in Semiconductors (Springer, US, 1964)

39. Rotenberg, E. \& Bostwick, A. microARPES and nanoARPES at diffractionlimited light sources: opportunities and performance gains. J. Synchrotron Radiat. 21, 1048-1056 (2014).

40. Kogar, A. et al. Surface collective modes in the topological insulators $\mathrm{Bi}_{2} \mathrm{Se}_{3}$ and $\mathrm{Bi}_{0.5} \mathrm{Sb}_{1.5} \mathrm{Te}_{3-x} \mathrm{Se}_{x}$. Phys. Rev. Lett. 115, 257402 (2015).

41. Yin, J. et al. Plasmonics of topological insulators at optical frequencies. NPG Asia Mater. 9, e425 (2017)

42. Homola, J. Surface Plasmon Resonance Based Sensors (Springer, Berlin, Heidelberg, 2006).

43. Sobota, J. A. et al. Direct optical coupling to an unoccupied dirac surface state in the topological insulator $\mathrm{Bi}_{2} \mathrm{Se}_{3}$. Phys. Rev. Lett. 111, 136802 (2013).

44. Spataru, C. D. \& Léonard, F. Fermi-level pinning, charge transfer, and relaxation of spin-momentum locking at metal contacts to topological insulators. Phys. Rev. B 90, 085115 (2014).

45. Kohn, W. \& Sherrington, D. Two kinds of bosons and Bose condensates. Rev. Mod. Phys. 42, 1-11 (1970).

46. Zenker, B., Fehske, H. \& Beck, H. Fate of the excitonic insulator in the presence of phonons. Phys. Rev. B 90, 195118 (2014).

47. Haug, H. \& Hanamura, E. Derivation of the two-fluid model for Bosecondensed excitons. Phys. Rev. B 11, 3317-3328 (1975).

48. Hanamura, E. \& Haug, H. Will a bose-condensed exciton gas be superfluid? Solid State Commun. 15, 1567-1570 (1974).

49. Kosterlitz, J. M. \& Thouless, D. J. Ordering, metastability and phase transitions in two-dimensional systems. J. Phys. C 6, 1181 (1973).

50. Leggett, A. J. Bose-Einstein condensation in the alkali gases: some fundamental concepts. Rev. Mod. Phys. 73, 307-356 (2001).

51. Lobb, C. J., Abraham, D. W. \& Tinkham, M. Theoretical interpretation of resistive transition data from arrays of superconducting weak links. Phys. Rev. B 27, 150-157 (1983).

\section{Acknowledgements}

This work was supported by National Science Foundation Grant DMR-1838532 and DMR-1710737. S.Y.S. was supported by National Science Foundation Grant DMR1832728. This research used the Molecular Foundry and the Advanced Light Source, which are US Department of Energy Office of Science User Facilities under Contract No. DE-AC02-05CH11231. H.F. acknowledges the US National Science Foundation Research Experiences for Undergraduates (REU) programme under Grant No. PHY-1560482. We acknowledge Alex Weber-Bargioni, Edward Bernard and Hans Bechtel at the Molecular Foundry for the assistance on optical measurements.

\section{Author contributions}

D.Y. and Y.H. designed the experiments. Y.H. synthesized $\mathrm{Bi}_{2} \mathrm{Se}_{3}$ nanoribbons and performed the measurements. R.X., L.M., H.C.T., J.F. and H.F. assisted the synthesis and measurements. A.R., I.V. and E.R. performed micro-ARPES measurements. R.W., O.E., B.W. and S.S. performed theoretical calculation. All the authors analysed the data. D.Y., Y.H., R.W., O.E. and I.V. co-wrote the paper.

\section{Competing interests}

The authors declare no competing interests.

\section{Additional information}

Supplementary information is available for this paper at https://doi.org/10.1038/s41467019-13711-3.

Correspondence and requests for materials should be addressed to D.Y.

Peer review information Nature Communications thanks Su-Yang $\mathrm{Xu}$ and the other, anonymous, reviewer(s) for their contribution to the peer review of this work. Peer reviewer reports are available.

Reprints and permission information is available at http://www.nature.com/reprints

Publisher's note Springer Nature remains neutral with regard to jurisdictional claims in published maps and institutional affiliations.

\begin{abstract}
(c) (i) Open Access This article is licensed under a Creative Commons Attribution 4.0 International License, which permits use, sharing, adaptation, distribution and reproduction in any medium or format, as long as you give appropriate credit to the original author(s) and the source, provide a link to the Creative Commons license, and indicate if changes were made. The images or other third party material in this article are included in the article's Creative Commons license, unless indicated otherwise in a credit line to the material. If material is not included in the article's Creative Commons license and your intended use is not permitted by statutory regulation or exceeds the permitted use, you will need to obtain permission directly from the copyright holder. To view a copy of this license, visit http://creativecommons.org/ licenses/by/4.0/.
\end{abstract}

(C) The Author(s) 2019 